Алгебра и анализ

Том. 18 (2006), № 4
St. Petersburg Math. J.

Vol. 18 (2007), No. 4, Pages 647-656 S 1061-0022(07)00964-8

Article electronically published on May 29, 2007

\title{
ESTIMATION OF A QUADRATIC FUNCTION AND THE $p$-BANACH-SAKS PROPERTY
}

\author{
E. M. SEMENOV AND F. A. SUKOCHEV
}

\begin{abstract}
Let $E$ be a rearrangement-invariant Banach function space on $[0,1]$, and let $\Gamma(E)$ denote the set of all $p \geq 1$ such that any sequence $\left\{x_{n}\right\}$ in $E$ converging weakly to 0 has a subsequence $\left\{y_{n}\right\}$ with $\sup _{m} m^{-1 / p}\left\|\sum_{1 \leq k \leq m} y_{n}\right\|<\infty$. The set $\Gamma_{i}(E)$ is defined similarly, but only sequences $\left\{x_{n}\right\}$ of independent random variables are taken into account. It is proved (under the assumption $\Gamma(E) \neq\{1\}$ ) that if $\Gamma_{i}(E) \backslash \Gamma(E) \neq \varnothing$, then $\Gamma_{i}(E) \backslash \Gamma(E)=\{2\}$.
\end{abstract}

$\S 1$

A classical Banach-Saks theorem (see [B, Chapter 12, Theorem 2]) states that if a sequence $x_{n} \in L_{p}[0,1], 1<p<\infty$, converges weakly to zero, then there exists a sequence $n_{k} \in \mathbb{N}$ and a number $C>0$ such that

$$
\left\|\sum_{k=1}^{m} x_{n_{k}}\right\|_{L_{p}} \leq C m^{\max (1 / 2,1 / p)}
$$

for all $m \in \mathbb{N}$ ( $\mathbb{N}$ denotes the set of positive integers). For $p \in(2, \infty)$ this estimate follows also from $\mathrm{KP}$. The exponent $\max (1 / 2,1 / p)$ is sharp. It suffices to consider the Rademacher system $x_{n}(t)=\operatorname{sign} \sin 2^{n} \pi t, n \in \mathbb{N}$, for $p \geq 2$ and any sequence of normalized $L_{p}$-functions with disjoint supports for $p \leq 2$.

This theorem leads to the following definitions (see [J, Be ). Let $E$ be a Banach space, and let $p \geq 1$. A bounded sequence $\left\{x_{n}\right\}$ in $E$ is called a $p$ - $B S$-sequence if there exists a subsequence $\left\{y_{n}\right\} \subset\left\{x_{n}\right\}$, such that

$$
\sup _{m \in \mathbb{N}} m^{-\frac{1}{p}}\left\|\sum_{k=1}^{m} y_{k}\right\|_{E}<\infty .
$$

We shall say that $E$ possesses the $p$ - $B S$-property and write $E \in B S(p)$ if any sequence converging weakly to zero contains a $p$ - $B S$-subsequence. Obviously, every Banach space possesses the $1-B S$-property. The set $\Gamma(E)=\{p: p \geq 1, E \in B S(p)\}$ is either $[1, \alpha]$ or $[1, \alpha)$, for some $\alpha \in[1, \infty]$. This set will be called the index set of the space $E$, and $\alpha$ is the Banach-Saks index of $E$; we write $\gamma(E)=\alpha$ if $\Gamma(E)=[1, \alpha]$ and $\gamma(E)=\alpha-0$ if $\Gamma(E)=[1, \alpha)$. A related notion was introduced in $\underline{\mathbf{R}}$, where the coordinate Orlicz spaces with the $p$ - $B S$-property were described.

The Banach-Saks theorem says that $\gamma\left(L_{p}\right)=\min (p, 2)$ for $1<p<\infty$. It is known also that $\gamma\left(L_{1}\right)=\gamma\left(L_{\infty}\right)=1$. In $l_{p}$, the sequences that converge weakly to zero have a

2000 Mathematics Subject Classification. Primary 46E30.

Key words and phrases. Rearrangement-invariant space, $p$-Banach-Saks property.

The first author was supported by RFBR (grant no. 05-01-00629) and by the "Universities of Russia" program (grant no. 04.01.051). The second author was supported by the Australia Research Counsil. 
relatively simple structure. By using these results, it is not hard to show that $\gamma\left(l_{p}\right)=p$ for $p \in(1, \infty), \gamma\left(l_{1}\right)=\gamma\left(c_{0}\right)=\infty$, and $\gamma\left(l_{\infty}\right)=1$.

A Banach space of measurable functions on $[0,1]$ is said to be rearrangement invariant (r.i.) or symmetric if

1) $|x(t)| \leq|y(t)|$ a.e. and $y \in E \Longrightarrow x \in E$ and $\|x\|_{E} \leq\|y\|_{E}$;

2) $x(t)$ and $y(t)$ are equimeasurable and $y \in E \Longrightarrow x \in E$ and $\|x\|_{E}=\|y\|_{E}$.

The theory of r.i. spaces is presented in the books [LT, [KPS]. The $p$ - $B S$-property in r.i. spaces was studied in [SS1, SS2, ASS, DSS]; in particular, there it was shown that the $p$ - $B S$-property is closely related to other geometric characteristics of r.i. spaces.

The index of any r.i. space does not exceed 2. Every nonseparable r.i. space has the trivial index 1 . Therefore, we shall study the $p$ - $B S$-property for separable r.i. spaces only.

Restricting ourselves, in the definitions of a $p$ - $B S$-sequence and the index set $\Gamma(E)$, to sequences of independent functions or to sequences of functions with disjoint supports, we arrive at the definitions of the sets $\Gamma_{i}(E)$ or $\Gamma_{d}(E)$, respectively. We recall that a system of functions $x_{i}(t)$ on $[0,1]$ is independent if

$$
\begin{gathered}
\operatorname{meas}\left\{t: t \in[0,1], x_{n}(t) \in\left(\alpha_{n}, \beta_{n}\right), n=1,2, \ldots, m\right\} \\
=\prod_{n=1}^{m} \operatorname{meas}\left\{t: t \in[0,1], x_{n}(t) \in\left(\alpha_{n}, \beta_{n}\right)\right\}
\end{gathered}
$$

for any $\alpha_{n}<\beta_{n}, n=1,2, \ldots, m$ and any $m \in \mathbb{N}$. The simplest and most important example of an independent system of functions is provided by the Rademacher system. Obviously, $\Gamma(E) \subset \Gamma_{i}(E)$ and $\Gamma(E) \subset \Gamma_{d}(E)$. In [ASS, L.4.1] it was shown that $\Gamma_{i}(E) \subset$ $\Gamma_{d}(E)$ for every separable r.i. space $E$.

As a rule, determining the index set $\Gamma(E)$ for a specific r.i. space is a fairly complicated task. Therefore, it is of interest to describe the set $\Gamma_{i}(E)$; this is much simpler, because of the known estimates of sums of independent functions (see [KS, Chapter 2]).

A large part of the paper [SS1] was devoted to the calculation of the index of the spaces $L_{p, q}$.

In the present paper, we aim at proving the following statement: either the set $\Gamma_{i}(E) \backslash \Gamma(E)$ is empty, or this set consists of one point and this point is 2. For this, we were bound to undertake a special study of a certain quadratic function generated by a system of measure-preserving transformations. Possibly, these auxiliary results are also of independent interest.

Lemma 1. If $r, j \in \mathbb{N}$ and $r \leq j$, then

$$
\sum_{k=1}^{j} \frac{\min (k r, j)}{k^{3 / 2}} \geq \frac{1}{2} \sqrt{j r} .
$$

Proof. Simple calculations show that for $r=1$ and $r=2$, inequality (10) is true with constant 1 (rather than $\frac{1}{2}$ ). This implies inequality (11) for $r \leq 8$. If $8 \leq r \leq j$, then

$$
\begin{aligned}
\sum_{k=1}^{j} \frac{\min (k r, j)}{k^{3 / 2}} & >\sum_{\frac{j}{r} \leq k \leq j} \frac{j}{k^{3 / 2}} \geq j \int_{\frac{j}{r}+1}^{j} \frac{d t}{t^{3 / 2}} \\
& =j\left(2\left(\frac{r}{j+r}\right)^{1 / 2}-2\left(\frac{1}{j}\right)^{1 / 2}\right) \geq \sqrt{j r}\left(\sqrt{2}-\frac{2}{\sqrt{r}}\right) \\
& \geq \sqrt{j r}\left(\sqrt{2}-\frac{1}{\sqrt{2}}\right)>\frac{1}{2} \sqrt{j r} .
\end{aligned}
$$


Let $k, m \in \mathbb{N}$. We denote by $\sigma_{k}$ the dilation operator that acts on $R^{m}$ as follows: if $x=\left(x_{1}, x_{2}, \ldots, x_{m}\right)$, then

$$
\sigma_{k} x=(\underbrace{x_{1}, x_{1}, \ldots, x_{1}}_{k}, \underbrace{x_{2}, x_{2}, \ldots, x_{2}}_{k}, \ldots) .
$$

In particular, for $k \geq m$ we have $\sigma_{k} x=\left(x_{1}, x_{1}, \ldots, x_{1}\right)$. If $x(t)$ is a function on $[0,1]$, we put $\sigma_{k} x(t)=x(t / k)$. It is well known that the operators $\sigma_{k}$ are bounded in every r.i. space.

For $x, y \in R^{m}$, the relation $x \prec y$ will mean that

$$
\sum_{i=1}^{j} x_{i}^{*} \leq \sum_{i=1}^{j} y_{i}^{*}
$$

for all $j=1,2, \ldots, m$, where $x_{i}^{*}$ is the monotone decreasing rearrangement of the numbers $\left|x_{i}\right|$.

Lemma 2. Suppose $x \in R^{m}, x_{1} \geq x_{2} \geq \cdots \geq x_{m} \geq 0$. Then

$$
\left\{\left(\frac{1}{k} \sum_{i=1}^{k} x_{i}^{2}\right)^{1 / 2}\right\}_{k=1}^{m} \prec 4 \sum_{k=1}^{m} \frac{\sigma_{k} x}{k^{3 / 2}} .
$$

Proof. First, we consider the particular case where

$$
x=x^{r}=(\underbrace{1,1, \ldots, 1}_{r}, \underbrace{0,0, \ldots, 0}_{m-r}), \quad 1 \leq r \leq m .
$$

Then

and

$$
\left(\frac{1}{k} \sum_{i=1}^{k} x_{i}^{2}\right)^{1 / 2}= \begin{cases}1, & k \leq r \\ \left(\frac{r}{k}\right)^{1 / 2}, & k \geq r\end{cases}
$$

for $j \leq r$, while

$$
\sum_{k=1}^{j}\left(\frac{1}{k} \sum_{i=1}^{k} x_{i}^{2}\right)^{1 / 2}=j
$$

$$
\begin{aligned}
& \sum_{k=1}^{j}\left(\frac{1}{k} \sum_{i=1}^{k} x_{i}^{2}\right)^{1 / 2}=r+\sum_{k=r+1}^{j}\left(\frac{r}{k}\right)^{1 / 2} \\
& \leq r+\sqrt{r} \int_{r}^{j} \frac{d t}{\sqrt{t}}=r+2 \sqrt{r}(\sqrt{j}-\sqrt{r})<2 \sqrt{j r}
\end{aligned}
$$

for $j>r$. Therefore, it suffices to show that

$$
j \leq 4 \sum_{i=1}^{j} \sum_{k=1}^{m} \frac{\left(\sigma_{k} x\right)_{i}}{k^{3 / 2}}
$$

for $j \leq r$, and

$$
\sqrt{j r} \leq 2 \sum_{i=1}^{j} \sum_{k=1}^{m} \frac{\left(\sigma_{k} x\right)_{i}}{k^{3 / 2}}
$$

for $j>r$. The obvious estimate

$$
\sum_{i=1}^{j} \sum_{k=1}^{m} \frac{\left(\sigma_{k} x\right)_{i}}{k^{3 / 2}}>\sum_{i=1}^{j} \sum_{k=1}^{m} \frac{1}{k^{3 / 2}}>\sum_{i=1}^{j} 1=j, \quad j \leq r,
$$


implies (3). Since

$$
\sum_{i=1}^{j}\left(\sigma_{k} x\right)_{i}=\min (k r, j)
$$

inequality (4) follows from Lemma 1, Thus, the inequality

$$
\sum_{k=1}^{j}\left(\frac{1}{k} \sum_{i=1}^{k} x_{i}^{2}\right)^{1 / 2} \leq 4 \sum_{i=1}^{j} \sum_{k=1}^{m} \frac{\left(\sigma_{k} x\right)_{i}}{k^{3 / 2}}
$$

is established for all $j=1,2, \ldots, m$ and all $x=x^{r}, 1 \leq r \leq m$.

Now, let $x \in R^{m}$, and let $1 \geq x_{1} \geq x_{2} \geq \cdots \geq x_{m} \geq 0$. The extreme points of this closed convex set are well known: these are the vectors $x^{r}, 0 \leq r \leq m$. There exists a sequence $\lambda_{0}, \lambda_{1}, \ldots, \lambda_{m}$, such that $\lambda_{r} \geq 0$ for all $r=0,1, \ldots, m, \sum_{r=0}^{m} \lambda_{r}=1$, and

$$
x=\sum_{r=0}^{m} \lambda_{r} x^{r} .
$$

We denote by $P=P_{j}$ and $Q=Q_{j}$ the operators occurring on the left and on the right side of (5). Since $P$ is convex and $Q$ is linear, we can use (5) to obtain

$$
\begin{aligned}
P x & =P\left(\sum_{r=0}^{m} \lambda_{r} x^{r}\right) \leq \sum_{r=0}^{m} \lambda_{r} P\left(x^{r}\right) \\
& \leq \sum_{r=0}^{m} \lambda_{r} 4 Q\left(x^{r}\right)=4 Q\left(\sum_{r=0}^{m} \lambda_{r} x^{r}\right)=4 Q x .
\end{aligned}
$$

This proves (5) under the additional restriction $x_{1} \leq 1$. Using positive homogeneity, we extend this estimate to all nonnegative $x \in R^{m}$ that are monotone nondecreasing.

Let $m, n \in \mathbb{N}$. We denote by $\Pi_{m}$ the set of all permutations of $\{1,2, \ldots, m\}$ and by $\Pi_{m, n}$ the set of all sequences $\left(\pi_{1}, \pi_{2}, \ldots, \pi_{n}\right)$, where $\pi_{1}, \pi_{2}, \ldots, \pi_{n} \in \Pi_{m}$. Given $\pi=\left(\pi_{1}, \pi_{2}, \ldots, \pi_{n}\right) \in \Pi_{m, n}$, we consider the operator

$$
\left(R_{\pi, m} x\right)_{i}=\left(\sum_{k=1}^{n} x_{\pi_{k}(i)}^{2}\right)^{1 / 2} .
$$

Lemma 3. Suppose $m, n \in \mathbb{N}, x \in R^{m}, x_{1} \geq x_{2} \geq \cdots \geq x_{m} \geq 0, \varepsilon>0$, and $\pi \in \Pi_{m, n}$. There exists a vector

$$
z \in \operatorname{conv}\left\{\left(\frac{n}{k}\right)^{1 / 2+\varepsilon} \sigma_{k} x, 1 \leq k \leq n\right\}
$$

such that $R_{\pi, m} x \prec \frac{4}{\varepsilon} z$.

Proof. There is no loss of generality in assuming that $x_{1}>x_{2}>\cdots>x_{m}>0$. The set

$$
\left\{(k, i): x_{\pi_{k}(i)} \geq x_{j}\right\}
$$

consists of $j n$ elements. Therefore,

$$
\left(R_{\pi, m} x\right)_{j}^{*} \leq\left(\frac{1}{j} \sum_{i=1}^{j} n x_{i}^{2}\right)^{1 / 2}=\left(\frac{n}{j} \sum_{i=1}^{j} x_{i}^{2}\right)^{1 / 2}
$$

for $1 \leq j \leq m$.

We have

$$
\sum_{k=1}^{n} \frac{\sigma_{k} x}{k^{3 / 2}}=\frac{1}{\varepsilon} \sum_{k=1}^{n} \frac{\varepsilon}{n^{\varepsilon} k^{1-\varepsilon}} \frac{n^{\varepsilon}}{k^{1 / 2+\varepsilon}} \sigma_{k} x
$$


and

$$
\sum_{k=1}^{n} \frac{\varepsilon}{n^{\varepsilon} k^{1-\varepsilon}} \leq \frac{\varepsilon}{n^{\varepsilon}} \int_{0}^{n} \frac{d t}{t^{1-\varepsilon}}=1 .
$$

Consequently, for some $\gamma \geq \varepsilon$ we have

$$
\gamma \sum_{k=1}^{n} \frac{\sigma_{k} x}{k^{3 / 2}} \in \operatorname{conv}\left\{\frac{n^{\varepsilon}}{k^{1 / 2+\varepsilon}} \sigma_{k} x, 1 \leq k \leq n\right\} .
$$

Put

$$
z=\gamma \sum_{k=1}^{n} \frac{\sigma_{k} x}{k^{3 / 2}} n^{1 / 2}
$$

Then

$$
z \in \operatorname{conv}\left\{\left(\frac{n}{k}\right)^{1 / 2+\varepsilon} \sigma_{k} x, 1 \leq k \leq n\right\} .
$$

Using (6) and Lemma 2, we obtain

$$
R_{\pi, m} x \prec \frac{4}{\varepsilon} z .
$$

Since the norm in any finite-dimensional space $F$ with symmetric basis is monotone relative to the partial ordering $\prec$ (see [LT, 2.a.8]), under the assumptions of Lemma 2 we have

$$
\begin{aligned}
\left\|R_{\pi, m} x\right\|_{F} & \leq\left\|\frac{4}{\varepsilon} z\right\|_{F} \leq \frac{4}{\varepsilon} \max _{1 \leq k \leq n}\left\|\left(\frac{n}{k}\right)^{1 / 2+\varepsilon} \sigma_{k} x\right\|_{F} \\
& \leq \frac{4}{\varepsilon} \max _{1 \leq k \leq n}\left(\frac{n}{k}\right)^{1 / 2+\varepsilon}\left\|\sigma_{k}\right\|_{F}\|x\|_{F} .
\end{aligned}
$$

Let $n \in \mathbb{N}$, and let $\omega=\left(\omega_{1}, \omega_{2}, \ldots, w_{n}\right)$ be a sequence of measure-preserving transformations of the segment $[0,1]$. The operator

$$
S_{n, \omega} x(t)=\left(\sum_{k=1}^{n} x^{2}\left(\omega_{k}(t)\right)\right)^{1 / 2}
$$

acts in any r.i. space $E$. The next lemma is usually viewed as routine. We present the proof for completeness.

Lemma 4. Suppose $n \in \mathbb{N}$,

$$
x(t)=\sum_{k=1}^{n} x_{k} æ_{\left(\frac{k-1}{n}, \frac{k}{n}\right)}(t)
$$

is a step function, $\omega$ is a measure-preserving transformation of $[0,1]$, and $T_{\omega} x(t)=$ $x(\omega(t))$. Then

$$
\lim _{m \rightarrow \infty, \frac{m}{n} \in \mathbb{N},} \min _{\pi \in \Pi_{m}} \operatorname{meas}\left\{t: T_{\omega} x(t) \neq \sum_{i=1}^{m} \bar{x}_{\pi(i)} \mathfrak{x}_{\left(\frac{i-1}{m}, \frac{i}{m}\right)}(t)\right\}=0,
$$

where $\bar{x}_{i}=x_{k}$ for $\frac{m}{n}(k-1)<i \leq \frac{m}{n} k, k=1,2, \ldots, n$.

Proof. Let $e_{k}$ denote the image of the segment $\left(\frac{k-1}{n}, \frac{k}{n}\right)$ under $\omega$, i.e., $æ_{e_{k}}=T_{\omega} æ_{\left(\frac{k-1}{n}, \frac{k}{n}\right)}$, $1 \leq k \leq n$. For a given $\varepsilon>0$, we find a system of disjoint intervals $\left(\alpha_{k, i}, \beta_{k, i}\right), i=$ $1,2, \ldots, s_{k}, k=1,2, \ldots, n$, such that

$$
\sum_{k=1}^{n} \operatorname{meas}\left(\left(e_{k} \backslash g_{k}\right) \cup\left(g_{k} \backslash e_{k}\right)\right)<\frac{\varepsilon}{2}
$$


where

$$
g_{k}=\bigcup_{i=1}^{s_{k}}\left(\alpha_{k, i}, \beta_{k, i}\right)
$$

We may assume that meas $g_{k} \leq$ meas $e_{k}$ for all $k=1,2, \ldots, n$ and that $g_{k} \cap g_{i}=\varnothing$ for $k \neq i$.

We choose $m \in \mathbb{N}$ so that $\frac{m}{n} \in \mathbb{N}$ and $m>\frac{2}{\varepsilon} \sum_{k=1}^{n} s_{k}$. Then the measure of the union of all intervals $\left(\frac{i-1}{m}, \frac{i}{m}\right), 1 \leq i \leq m$, that contain at least one point of the set $\left\{\alpha_{k, i}, \beta_{k, i}, 1 \leq i \leq s_{k}, k=1,2, \ldots, n\right\}$ is less than $\frac{\varepsilon}{2}$. Put

$$
A_{k}=\left\{i: i \in \mathbb{N},\left(\frac{i-1}{m}, \frac{i}{m}\right) \subset g_{k}\right\}, \quad k=1,2, \ldots, n .
$$

Then

$$
\left|A_{k}\right| \leq\left|\left\{i:\left(\frac{i-1}{m}, \frac{i}{m}\right) \subset\left(\frac{k-1}{n}, \frac{k}{n}\right)\right\}\right|=\frac{m}{n}
$$

for all $k=1,2, \ldots, n$. The set $\left\{i: \frac{m}{n}(k-1)<i \leq \frac{m}{n} k\right\}$ consists of $\frac{m}{n}$ elements. Therefore, there exists a permutation $\pi \in \Pi_{m}$ such that $\pi^{-1}(i) \in\left(\frac{m}{n}(k-1), \frac{m}{n} k\right]$ for all $i \in A_{k}$, $k=1,2, \ldots, n$. For this $\pi \in \Pi_{m}$, we have

$$
\text { meas }\left\{t: T_{\omega} x(t) \neq \sum_{i=1}^{m} \bar{x}_{\pi(i)} æ_{\left(\frac{i-1}{m}, \frac{i}{m}\right)}(t)\right\} \leq \frac{\varepsilon}{2}+\frac{\varepsilon}{2}=\varepsilon .
$$

Given an r.i. space $E$ and $m \in \mathbb{N}$, we can construct an $m$-dimensional space $E_{m}$ with symmetric basis: if $x=\left(x_{1}, x_{2}, \ldots, x_{m}\right)$, then

$$
\|x\|_{E_{m}}=\left\|\sum_{k=1}^{m} x_{k} æ_{\left(\frac{k-1}{m}, \frac{k}{m}\right)}\right\|_{E} .
$$

Lemma 5. Let $E$ be a separable r.i. space. Then

$$
\left\|S_{n, \omega}\right\|_{E} \leq \sup _{m \in \mathbb{N}, \pi \in \Pi_{m}}\left\|R_{\pi, m}\right\|_{E_{m}} .
$$

Proof. Since $E$ is separable, for any $\varepsilon>0$ we can find a step function

$$
x(t)=\sum_{k=1}^{r} x_{k} æ_{\left(\frac{k-1}{r}, \frac{k}{r}\right)}(t),
$$

such that $\|x\|_{E}=1$ and

$$
\left\|S_{n, \omega} x\right\|_{E} \geq\left\|S_{n, \omega}\right\|_{E}-\frac{\varepsilon}{2} .
$$

The structure of the operator $S_{n, \omega}$ allows us to assume that $x_{1} \geq x_{2} \geq \cdots \geq x_{r} \geq 0$. In a separable r.i. space, the norm of each function is absolutely continuous, i.e.,

$$
\lim _{\delta \rightarrow 0} \sup _{\text {meas } e \leq \delta}\left\|x æ_{e}\right\|_{E}=0 .
$$

Thus, there exists $\delta>0$ such that $\left\|x æ_{e}\right\|_{E}<\frac{\varepsilon}{4 n}$ whenever meas $e<\delta$. By Lemma 4, there are $m \in \mathbb{N}$ and $\pi=\left(\pi_{1}, \pi_{2}, \ldots, \pi_{n}\right) \in \Pi_{m, n}$ such that

$$
\text { meas }\left\{t: T_{\omega_{k}} x(t) \neq \sum_{i=1}^{m} \bar{x}_{\pi_{k}(i)} \Re_{\left(\frac{i-1}{m}, \frac{i}{m}\right)}(t)\right\}<\delta
$$

for all $k=1,2, \ldots, n$. The functions $T_{\omega_{k}} x$ and

$$
\sum_{i=1}^{m} \bar{x}_{\pi_{k}(i)}\left(_{\left(\frac{i-1}{m}, \frac{i}{m}\right)}\right.
$$


are equimeasurable with $x$ for all $k=1,2, \ldots, n$. Therefore,

$$
\left\|T_{\omega_{k}} x-P_{k} x\right\| \leq 2 \sup _{\text {meas } e \leq \delta}\left\|x æ_{e}\right\| \leq \frac{\varepsilon}{2 n}
$$

for all $k=1,2, \ldots, n$, where

$$
P_{k} x(t)=\sum_{i=1}^{m} \bar{x}_{\pi_{k}(i)} æ_{\left(\frac{i-1}{m}, \frac{i}{m}\right)}(t) .
$$

Then

$$
\begin{aligned}
& \left\|S_{n, \omega} x-\left(\sum_{k=1}^{n}\left(P_{k} x\right)^{2}\right)^{1 / 2}\right\|_{E} \\
& =\left\|\left(\sum_{k=1}^{n}\left(T_{\omega_{k}} x\right)^{2}\right)^{1 / 2}-\left(\sum_{k=1}^{n}\left(P_{k} x\right)^{2}\right)^{1 / 2}\right\|_{E} \\
& \leq\left\|\left(\sum_{k=1}^{n}\left(T_{\omega_{k}} x-P_{k} x\right)^{2}\right)^{1 / 2}\right\|_{E} \\
& \leq \sum_{k=1}^{n}\left\|T_{\omega_{k}} x-P_{k} x\right\|_{E} \leq \sum_{k=1}^{n} \frac{\varepsilon}{2 n}=\frac{\varepsilon}{2} .
\end{aligned}
$$

By (9) and (11), we have

$$
\left\|S_{n, \omega}\right\|_{E} \leq\left\|\left(\sum_{k=1}^{n}\left(P_{k} x\right)^{2}\right)^{1 / 2}\right\|_{E}+\varepsilon .
$$

If $\pi$ is the permutation defined in (10), then

$$
\left\|\left(\sum_{k=1}^{n}\left(P_{k} x\right)^{2}\right)^{1 / 2}\right\|_{E} \leq\left\|R_{\pi, m} x\right\|_{E} \leq\left\|R_{\pi, m}\right\|_{E} .
$$

The last two estimates imply (8).

It is clear that $\left\|\sigma_{k}\right\|_{E_{m}} \leq\left\|\sigma_{k}\right\|_{E}$ for all $k, m \in \mathbb{N}$, and that the norm of the operator $S_{n, \omega}$ is attained on the set of nonnegative, monotone nondecreasing functions. Therefore, (7) and Lemma 6 imply the following statement.

Theorem 6. If $E$ is a separable r.i. space, $n \in \mathbb{N}$ and $\varepsilon>0$, then

$$
\left\|S_{n, \omega}\right\|_{E} \leq \frac{4}{\varepsilon} \max _{1 \leq k \leq n}\left(\frac{n}{k}\right)^{1 / 2+\varepsilon}\left\|\sigma_{k}\right\|_{E} .
$$

If $E$ is the Lorentz space $L_{2,1}$, then $\left\|\sigma_{k}\right\|_{E}=k^{1 / 2}$ for all $k \in \mathbb{N}$, and

$$
\lim _{n \rightarrow \infty}\left\|S_{n, \omega}\right\|_{E} \cdot n^{-1 / 2}=\infty
$$

for some sequence $\omega$. This shows that the estimate

$$
\left\|S_{n, \omega}\right\|_{E} \leq C \max _{1 \leq k \leq n}\left(\frac{n}{k}\right)^{1 / 2}\left\|\sigma_{k}\right\|_{E},
$$

with $C$ independent of $n$, cannot be obtained. 
Now we pass to the proof of the main result of the paper. If $E$ is an r.i. space, we denote by $E^{\prime \prime}$ the set of $x \in L_{1}[0,1]$ such that

$$
\|x\|_{E^{\prime \prime}}=\sup _{n}\|\min (|x(t)|, n)\|_{E}<\infty .
$$

The inclusion $E \subset E^{\prime \prime}$ is well known to be isometric for any separable r.i. space $E$. The space $E^{\prime \prime}$ either coincides with $E$ or is nonseparable.

Theorem 7. Suppose $1<p<2, E$ is a separable r.i. space on $[0,1], \Gamma(E) \neq\{1\}$, and $p \in \Gamma_{i}(E)$. Then $p \in \Gamma(E)$.

Proof. Let $x_{n} \in E$ be such that $\left\|x_{n}\right\|_{E} \leq 1$ for all $n \in \mathbb{N}$. Suppose that the $x_{n}$ converge weakly to zero in $E$. By ASS, Lemma 3.6], there is a subsequence $\left\{y_{n}\right\} \subset\left\{x_{n}\right\}$, a function $u \in E^{\prime \prime}$, and sequences of functions $u_{n}, v_{n}, w_{n} \in E$, such that $y_{n}=u_{n}+v_{n}+w_{n}$, $u_{n}^{*} \leq u,\|u\|_{E} \leq 1$, the $v_{n}$ are pairwise disjoint, $\left\|v_{n}\right\|_{E} \leq 2$ for $n \in \mathbb{N}$, and $\lim _{n \rightarrow \infty}\left\|w_{n}\right\|=$ 0 .

By [ASS, Lemma 4.1(ii)], we have $p \in \Gamma_{d}(E)$. Therefore, there is a constant $C_{1}>0$ and a subsequence $v_{i_{n}}$ such that

$$
\left\|\sum_{i=1}^{m} v_{i_{n}}\right\|_{E} \leq C_{1} m^{1 / p} \sup _{n}\left\|v_{i_{n}}\right\|_{E} \leq 2 C_{1} m^{1 / p}
$$

for all $m \in \mathbb{N}$. To simplify the notation, we assume that $i_{n}=n$.

In Theorem 4.3 of [SS1] (which may be applied due to [ASS, Theorem 4.2(i)]), it was proved that there exists a constant $C_{2}>0$ such that

$$
\left\|\sum_{n=1}^{m} u_{n}\right\|_{E} \leq C_{2}\left\|\left(\sum_{n=1}^{m} u_{n}^{2}\right)^{1 / 2}\right\|_{E}+1
$$

for all $m \in \mathbb{N}$. Since $u_{n}^{*} \leq u$, we have $u_{n} \leq u\left(\omega_{n}\right)$, where $\omega_{n}$ is a measure-preserving transformation of $[0,1], n \in \mathbb{N}$. Therefore,

$$
\left\|\sum_{n=1}^{m} u_{n}\right\|_{E} \leq C_{2}\left\|S_{m, \omega} u\right\|_{E^{\prime \prime}}+1
$$

where $\left(\omega=\omega_{1}, \omega_{2}, \ldots, \omega_{m}\right), m \in \mathbb{N}$. The function $u$ defined in ASS, Lemma 3.6] belongs to $E^{\prime \prime}$, but may fail to belong to $E$. However, when estimating $\left\|\sum_{n=1}^{m} u_{n}\right\|$, we may replace $u$ with the function

$$
v(t)=\min \left(u^{*}(t), \max _{1 \leq n \leq m} u_{n}^{*}(t)\right) .
$$

Since $v \in E$ and the inclusion $E \subset E^{\prime \prime}$ is isometric, there is no loss of generality in assuming that $u \in E$ and

$$
\left\|\sum_{n=1}^{m} u_{n}\right\|_{E} \leq C_{2}\left\|S_{m, \omega} u\right\|_{E}+1 .
$$

Now we apply Theorem 6, putting $\varepsilon=\frac{1}{p}-\frac{1}{2}$. Then

$$
\left\|\sum_{n=1}^{m} u_{n}\right\|_{E} \leq C_{2} \frac{2 p}{2-p} \cdot 4 \max _{1 \leq k \leq m}\left(\frac{m}{k}\right)^{\frac{1}{p}}\left\|\sigma_{k}\right\|_{E}+1 .
$$


Since $p \in \Gamma_{i}(E)$, the proof of [ASS, Theorem 4.2(ii)] shows that there exists a constant $C_{3}>0$ such that $\left\|\sigma_{k}\right\|_{E} \leq C_{3} k^{1 / p}$ for all $k \in \mathbb{N}$. Consequently,

$$
\left\|\sum_{n=1}^{m} u_{n}\right\|_{E} \leq \frac{16 C_{2}}{2-p} \max _{1 \leq k \leq m}\left(\frac{m}{k}\right)^{\frac{1}{p}} C_{3} k^{1 / p}+1=\frac{16 C_{2} C_{3}}{2-p} m^{1 / p}+1
$$

for all $m \in \mathbb{N}$.

As was mentioned above, $\lim _{n \rightarrow \infty}\left\|w_{n}\right\|_{E}=0$. Passing to a subsequence, we may assume that

$$
\sum_{n=1}^{\infty}\left\|w_{n}\right\|_{E} \leq 1
$$

Combining (12), (13), and (14), we see that there exists a subsequence $\left\{x_{j_{n}}\right\} \subset\left\{x_{n}\right\}$ and a constant $C_{4}>0$ such that

$$
\left\|\sum_{n=1}^{m} x_{j_{n}}\right\|_{E} \leq C_{4} m^{1 / p}
$$

for all $m \in \mathbb{N}$. We can take $C_{4}=2 C_{1}+\frac{16 C_{2} C_{3}}{2-p}+2$. This means that $p \in \Gamma(E)$.

Thus,

$$
\Gamma(E) \cap[1,2)=\Gamma_{i}(E) \cap[1,2) .
$$

In the proof of Proposition 4.12 in [ASS and in Theorem 5.9 of [SS1] it was shown that $\Gamma_{i}\left(L_{2,1}\right)=[1,2]$ and $\Gamma\left(L_{2,1}\right)=[1,2)$. This tells us that Theorem 7 does not extend to the case of $p=2$. However, if $2 \in \Gamma_{i}(E)$, then $p \in \Gamma_{i}(E)$ for any $p \in[1,2)$. We arrive at the following consequence of Theorem 7 .

Corollary 8. If $E$ is a separable r.i. space such that $\Gamma(E) \neq\{1\}, 2 \in \Gamma_{i}(E)$, and $1<p<2$, then $p \in \Gamma(E)$.

Lemma 4.1 in $\left[\mathrm{ASS}\right.$ ] states that $1 \in \Gamma(E) \subset \Gamma_{i}(E) \subset[1,2]$ for any separable r.i. space $E$. Combining this with Theorem 7 and Corollary 8 , we see that the following is true.

Theorem 9. Let $E$ be a separable r.i. space with $\Gamma(E) \neq\{1\}$. Then either (i) $\Gamma(E)=$ $\Gamma_{i}(E)$ or (ii) $\Gamma(E)=[1,2)$ and $\Gamma_{i}(E)=[1,2]$.

We note that, in contrast to $\Gamma_{i}(E)$, the set $\Gamma_{d}(E)$ may differ significantly from $\Gamma(E)$. For example, if $E$ is a separable Lorentz space, then $\Gamma_{d}(E)=[1, \infty)$ and $\Gamma(E) \subset \Gamma_{i}(E) \subset$ $[1,2]$.

\section{REFERENCES}

[ASS] S. B. Astashkin, E. M. Semenov, and F. A. Sukochev, The Banach-Saks p-property, Math. Ann. 332 (2005), 879-900. MR2179781 (2006h:46028)

[B] S. Banach, Theory of linear operations, North-Holland Math. Library, vol. 38, North-Holland Publ. Co., Amsterdam, 1987. MR880204 (88a:01065)

[Be] B. Beauzamy, Banach-Saks properties and spreading models, Math. Scand. 44 (1979), 357-384. MR555227 (81a:46018)

[DSS] P. G. Dodds, E. M. Semenov, and F. A. Sukochev, The Banach-Saks property in rearrangement invariant spaces, Studia Math. 162 (2004), 263-294. MR2047655 (2004m:46006)

[J] W. B. Johnson, On quotients of $L_{p}$ which are quotients of $l_{p}$, Compositio Math. 34 (1977), 69-89. MR0454595 (56:12844)

[KP] M. I. Kadec and A. Pelczyński, Bases, lacunary sequences and complemented subspaces in the spaces $L_{p}$, Studia Math. 21 (1961/1962), 161-176. MR0152879(27:2851)

[KPS] S. G. Kreĭn, Yu. I. Petunin, and E. M. Semenov, Interpolation of linear operators, "Nauka", Moscow, 1978; English transl., Transl. Math. Monogr., vol. 54, Amer. Math. Soc., Providence, RI, 1982. MR506343 (81f:46086), MR0649411(84j:46103) 
[KS] B. S. Kashin and A. A. Saakyan, Orthogonal series, "Nauka", Moscow, 1984; English transl., Transl. Math. Monogr., vol. 75, Amer. Math. Soc., Providence, RI, 1989. MR0779286 (86k:42046) MR:1007141 (90g:42001)

[LT] J. Lindenstrauss and L. Tzafriri, Classical Banach spaces. II. Function spaces, Ergeb. Math. Grenzgeb., vol. 97, Springer-Verlag, Berlin-New York, 1979. MR540367 (81c:46001)

[R] S. A. Rakov, The Banach-Saks exponent of some Banach spaces of sequences, Mat. Zametki 32 (1982), no. 5, 613-625; English transl., Math. Notes 32 (1982), no. 5-6, 791-797 (1983). MR684604 (84c:46018)

[SS1] E. M. Semenov and F. A. Sukochev, The Banach-Saks index, Mat. Sb. 195 (2004), no. 2, 117-140; English transl., Sb. Math. 195 (2004), no. 1-2, 263-285. MR2068953 (2005d:46063)

[SS2] - The Banach-Saks index of rearrangement invariant spaces on [0,1], C. R. Math. Acad. Sci. Paris. 337 (2003), 397-401. MR2015083 (2004h:46028)

Voronezh State University, Universitetskaya Ploshchad ${ }^{\prime}$ 1, 394007 Voronezh, Russia

E-mail address: semenov@func.vsu.ru

Flinders University of South Australia, Bedford Park, 5042, SA, Australia

E-mail address: sukochev@infoeng.flinders.edu.au

Received 22/FEB/2006

Translated by A. PLOTKIN 